\title{
Lattice Boltzmann modeling of injection moulding process
}

\author{
Jonas Latt ${ }^{1}$, Guy Courbebaisse ${ }^{2}$, Bastien Chopard ${ }^{1}$, and Jean Luc Falcone ${ }^{1}$ \\ 1 University of Geneva, Computer Science Department, 1211 Geneva 4, Switzerland, \\ Bastien.Chopard@cui.unige.ch, \\ WWW home page: http://cui.unige.ch/ ${ }^{\sim}$ chopard/home.html \\ 2 LRPP - ERT 10 - ESP, 85 Rue Henri Becquerel \\ F-01100 Bellignat, France \\ courbebaisse@free.fr
}

\begin{abstract}
Polymer injection in moulds with complicated shapes is common in todays industrial problems. A challenge is to optimize the mould design which leads to the most homogeneous filling. Current commercial softwares are able to simulate the process only partially. This paper proposes a preliminary study of the capability of a two-fluid LatticeBoltzmann model to provide a simple and flexible approach which can be easily parallelized, will include correct contact angles and simulate the effect of the positioning of air-holes.
\end{abstract}

Keywords: Polymer injection moulding, Hele-Shaw model, mathematical morphology, lattice Boltzmann method, multi-component fluids, numerical simulation.

\section{Introduction}

In the injection moulding process, as in many other scientific problems, numerical simulations play an important role. Polymer injection pressure, injection points and locations of air evacuation channels all affect the quality of the final object. In particular, the re-attachment of two fronts of matter (weld lines) leads to strong internal constraints and weakens its mechanical resitance.

Commercial softwares, such as MoldFlow, offer powerful tools to simulate the moulding process in the free surface approximation. They provide a quantitative description in which polymer properties can be adjusted. However, they offer little flexibility to combine the simulation with a shape optimization procedure. In addition, the free surface approximation does not allow the designer to study the effect of the location of air evacuation points. Finally, parallelized versions of such softwares are often not available.

There is a need among the practitioners of this field to develop new approaches. In particular, there seems to be a lack of tools offering a pre-modeling of the injection process, with enough flexibility to investigate the impact of geometrical factors. There are several examples in which the mould geometry is 
dominating in comparison with the fluid rheological properties. For instance, a recent original method based solely on the propagation of distances demonstrates that the injection process is more driven by the geometry of the mould cavity than by the non-Newtonian features of the polymer. In other words, the crucial hydrodynamic properties are mass conservation, forbidden regions and behaviour at the mould boundaries.

Along this line, the use of mathematical morphology concepts $[1,2]$ for the simulation of the propagation of matter in a cavity, with one or more points of injection [3], is quite promising. The work by G. Aronsson [4] provides an explicit link between the concept of propagation of distances and the spreading of fluids with power-law stress tensors.

The goal of this paper is to exploit the flexibility and simplicity of the Lattice Boltzmann (LB) method [5] to address the mould injection process at a premodel level. We consider the so-called Shan-Chen model [6] of two immiscible fluids representing the polymer and air respectively. We assume that Newtonian fluids will provide good approximations if geometrical factors are dominant. In this preliminary study we shall not pay too much attention to the actual ratio of densities and viscosities of the two fluids.

We will first test our approach on the so-called Hele-Shaw situation (see for instance [7]) and then consider a more realistic mould cavity. The LB results are compared with MoldFlow simulations. A discussion of the critical features of our approach is finally given.

Note that, at the time of writing, we became aware of a similar work by Ginzburg [8] in which a free-surface LB model is developed and applied to the filling of a cavity. However, this model does not include air behaviour and does not take into account the effect of air evacuation points.

\section{The lattice Boltzmann model}

Lattice Boltzmann (LB) models are historically derived from cellular automata fluid models $[9,5]$. These models are seen as an alternative to finite element techniques for fluid flow computations. They have been successfully applied to multi-phase and multi-component flows and several other applications. In the present context, we propose to adapt the LB methods to the filling phase of an injection moulding process.

\subsection{The lattice Boltzmann equation for a single fluid}

A LB model is built on a discrete space-time universe. The fluid is described by density distributions function $f_{k}(\boldsymbol{x}, t), k=0 \ldots z$ containing the amount of fluid that enters lattice node $\boldsymbol{x}$ at time $t$, with velocity $\boldsymbol{v}_{k}, k=0 \ldots z$. The possible velocities are constrained by the lattice topology. Typically, $z$ is the lattice coordination number and the discrete set of velocities is chosen so that, in one time step $\Delta t$, the particles travel one lattice spacing $\Delta x$ in any of the $z$ 
lattice directions. One also considers a rest population of fluid particles $f_{0}$, with velocity $\boldsymbol{v}_{0}=0$.

The usual physical quantities such as local density and velocity are obtained from the $f_{k}$ by the following relations:

$$
\rho=\sum_{k=0}^{z} f_{k} \quad \text { and } \quad \boldsymbol{u}=\sum_{k=0}^{z} f_{k} \boldsymbol{v}_{k} .
$$

During time evolution, the fluid density functions are streamed on the lattice, in the direction specified by $k$. After the streaming, a collision occurs between all the $f_{k}$ entering the same lattice site. The result of this collision is to redistribute mass and momentum to each lattice directions, thus creating the post collision values of the $f_{k}$. It can be shown that, provided that the collision operator is properly chosen, the LB dynamics solves the Navier-Stokes equation $[9,5]$, in the low Mach number regime.

It is also shown that the fluid pressure is related to the fluid density by the ideal gas state equation $p=c_{s}^{2} \rho$, where $c_{s}^{2}$ is the speed of sound. Therefore, in a LB model, there is no need to solve the pressure equation. It is all built-in in the equation of motion for the $f_{k}$.

In what follows, we consider a two-dimensional system, with $z=8$. This corresponds to the so-called D2Q9 model [5]. The collision is obtained by a relaxation with coefficient $\omega$ to a truncated Maxwell-Boltzmann local equilibrium distribution function $f^{(e q)}$, which depends only on the current local fluid density $\rho$ and velocity $\boldsymbol{u}$. This approach is referred to as a LBGK model $[9,5]$.

The following equation summarizes the evolution rule:

$$
f_{i}\left(\boldsymbol{x}+\boldsymbol{v}_{i} \Delta t, t+\Delta t\right)-f_{i}(\boldsymbol{x}, t)=-\omega\left(f_{i}-f_{i}^{(e q)}\right)+\gamma \boldsymbol{F}_{\text {int }} \cdot \boldsymbol{v}_{i}
$$

where $F_{\text {int }}$ is a term which may account for extra interaction force acting on the particle density distribution. The coefficient $\gamma$ is a normalization parameter which depends on the chosen lattice topology.

In order to model a system of two immiscible fluids (polymer and air) we use the so-called Shan-Chen model [6]. Two sets of $f_{k}$ are defined, one for each species. The values corresponding to the two fluids will be distinguished by a supperscript $\sigma \in 0,1$. Each fluid follows the dynamics of a single fluid system, as given in eq 2 . There are only two changes that must be made to describe the interaction between the fluids.

First, the fluids define a common velocity and use it for the computation of the equilibrium distribution in equation 2

$$
\boldsymbol{u}=\boldsymbol{u}^{(0)}+\boldsymbol{u}^{(1)}=\frac{\sum_{\sigma, i} f_{i}^{\sigma} \boldsymbol{v}_{i}}{\sum_{\sigma, i} f_{i}^{\sigma}}
$$

Second, the term $F_{\text {int }}$ is computed so as to produce a repulsive interaction force between the two fluids. It depends on a parameter $W$ that accounts for the surface tension of the fluids:

$$
\boldsymbol{F}_{\text {int }}^{(\sigma)}(\boldsymbol{x}, t)=W \rho^{(\sigma)} \sum_{k} \Psi^{(\sigma)}\left(\boldsymbol{x}+\tau \boldsymbol{v}_{k}\right) \boldsymbol{v}_{k} .
$$


In the bulk of the fluid, the quantity $\Psi$ corresponds to the density of the other fluid:

$$
\Psi^{(\sigma)}=\rho^{(1-\sigma)} .
$$

When one models immiscible fluids, one chooses an interaction force that is sufficiently strong to keep the penetration between the fluids low. The simulated flow field is thus separated in two regions, in each of which one fluid is dominant. In a small range around the interface between those two regions, both fluids coexist symmetrically. In this range, the density variations are large and the approximation for an incompressible fluid is not valid any more. The results must rather be understood as representative of some kind of interface dynamics between the immiscible fluids.

\section{Boundary conditions}

On the boundaries of the domain, a specific dynamics is applied on the fluid density distributions $f_{i}^{\sigma}$ in order to produce the desired effect: no-slip on a wall, fixed air pressure on the air-holes and specific contact angle at the interface between air, polymer and solid walls. Finally, polymer injection points also need a special treatment. The boundary conditions we propose are discussed below.

\subsection{Bounce back boundaries}

We implement no-slip boundaries (i.e. boundaries on which the velocity is zero) by assigning bounce back dynamics to the boundary nodes. Thus, the dynamics (2) is replaced by a reflection rule:

$$
f_{i}^{(\sigma)}(\boldsymbol{x}, t+\Delta t)=f_{\text {oppositeOf }(i)}^{(\sigma)}(\boldsymbol{x}, t) .
$$

The index oppositeOf $(i)$ is the index associated with the direction opposite to direction $i$ : $v_{\text {opposite } O f(i)}=-v_{i}$.

The flow sites next to the boundaries interact with the boundary sites through a wetting parameter $g^{(\sigma)}$. The value of $\Psi$ from equation 4 is thus defined on the boundary nodes by

$$
\Psi^{(\sigma)}=g^{(\sigma)}
$$

The value of $g^{(\sigma)}$ determines the contact angle of the fluid interface on the wall [10]. In our simulations, we have chosen $g^{(\sigma)}=-g^{(1-\sigma)}$. The situation is explained on Figure 1. On this figure, fluid 1 is wetting, and fluid 0 is non-wetting. This effect is obtained by chosing $g^{(0)}>0$ and $g^{(1)}<0$.

\subsection{Inlet and outlet boundaries.}

As a boundary condition for the injection spot and the air-holes, we define semipermeable walls: one fluid is retained by a normal no-slip condition, while the other fluid is injected or evacuated. 
Fig. 1. Bounce back boundaries with a defined contact angle.

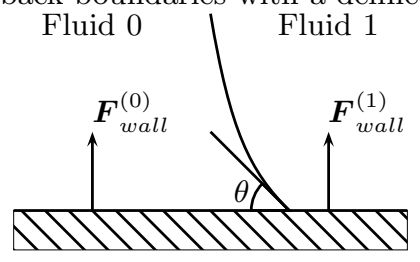

Let us consider as an example the case of fluid 0 being evacuated through the semipermeable wall. We chose to perform the evacuation at a constant pressure. This is achieved by applying on fluid 0 the (constant pressure) outlet boundary condition described in the reference [11]. Furthermore, an interaction term $\Psi^{(1)}=$

$\rho^{(0)}$ is defined on the wall. This means that fluid 1 acts like a bulk node (5), rather than a wall node $(6)$.

For fluid 1 we implement a (no-slip) bounce-back dynamics on the wall. Consistently with the presence of this semi-permeable wall, a wetting parameter $\Psi^{(0)}=g^{(0)}$ is defined.

\section{Simulation of an injection in free space}

A first validation of the numerical model is obtained from a simple simulation with cylindrical symmetry. A polymer is injected at a spot in free space and expands in the form of a disk of increasing radius $R$. This is the well known Hele-Shaw model [7]. In this case, the boundary conditions have a subsidiary importance. We can therefore verify whether the basic properties of the polymer are properly resolved by the model.

\subsection{Assumptions about material properties}

In the particular case of injection moulding, we consider only very high viscous fluids. In consequence, the inertial and gravitational terms will not be taken into account in the momentum equation. This simplification leads to a well known equation : the Stokes equation (see for instance [7]). During the filling phase, the polymer is assumed to be incompressible. The resulting equations are :

$$
\begin{gathered}
\nabla \cdot \boldsymbol{v}=0 \\
-\nabla p+\nabla \cdot \underline{\sigma}_{n}=0
\end{gathered}
$$

where $\boldsymbol{v}$ is the fluid velocity, $p$ the pressure and $\underline{\sigma}_{n}$ the viscous strain tensor. 


\subsection{Hele-Shaw assumptions}

The Hele-Shaw model describes the filling of a mould cavity whose thickness can be neglected. The filling fluid flow is modelled according to the Stokes equation between two plates separated by a short distance $h$.

We consider a cartesian coordinate system where the $z$-axis is in the thickness direction. The $x-y$ plane is on the mid plane of the cavity and the velocity components $u_{x}, u_{y}$ and $u_{z}$ are respectively taken in the $x, y$ and $z$ directions. The Hele-Shaw flow model consists of the following assumptions [12]:

- The $z$-component of the velocity is neglected with respect to the other velocity components.

- The pressure is constant across the thickness. It only depends on $x$ and $y$.

- The velocity gradient in the $x$ and $y$ directions is independent of the $z$ direction.

Applying the Hele-Shaw assumptions to the Stokes equation, one obtains the simplified expressions:

$$
\frac{\partial p}{\partial x}=\frac{\partial}{\partial z}\left(\eta \frac{\partial u_{x}}{\partial z}\right), \frac{\partial p}{\partial y}=\frac{\partial}{\partial z}\left(\eta \frac{\partial u_{y}}{\partial z}\right), \frac{\partial p}{\partial z}=0
$$

with $\eta$ the viscosity.

These equations can be solved explicitly in the case of a disk mould with a constant injection rate $Q[7]$.

Inside the injected polymer, the distribution of the pressure is predicted as follows:

$$
p(r)=a+b \ln r
$$

where $r<R$ is the distance to the injection point and $a$ and $b$ are constant. The distribution of the velocity is given by

$$
\rho(r) v(r)=\frac{Q}{2 \pi r}
$$

Finally, the expression for the surface growth of the polymer follows directly from the mass conservation in an incompressible fluid:

$$
\pi R^{2}=Q t
$$

\subsection{Numerical result}

The geometry of the simulation is a square domain of size $201 \times 201$, initially filled with air (fluid 1) at a density (pressure) of 1.0. At the boundary of the domain, the pressure of fluid 1 is kept constant at this value.

Fluid 1 (the polymer) is injected in the middle of the system, on one lattice site, with fixed density (pressure) $\rho_{1}=1.1$. The two fluids have same viscosity (relaxation coeficient $\omega=1$ ) and their mutual interaction is defined through $W=-0.3$. 


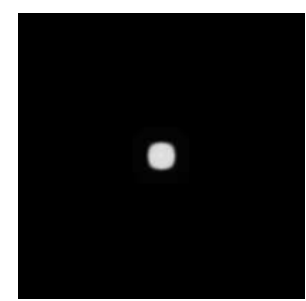

$t=4000$

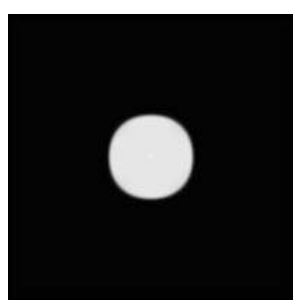

$t=40000$

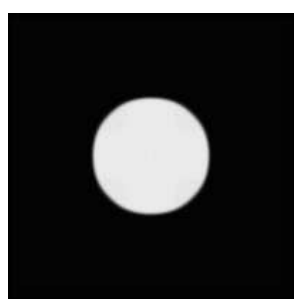

$t=80000$

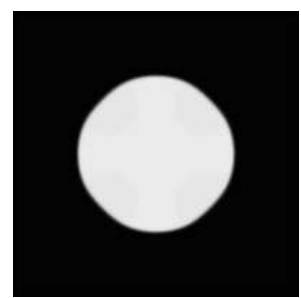

$t=140000$

Fig. 2. Amount of polymer injected as a function of time, in a free space. In the later stage, the anisotropy is due to the air which cannot escape fast enough out of the boundaries.

The evolution of the system is represented at four chosen time steps in Figure 2. We expect from equation 13 that the radius of the injected disk increases as a square root of the time. This fact is verified on the simulations, both from the program MoldFlow and from the lattice Boltzmann model (see Figure 3).

We have measured the velocity and pressure profile in the simulation at the time step $t=140000$. The results inside a radius of $r<30$ are shown in Figure 4 . They exclude the domain close to the free boundary that obeys special interface dynamics. Note that the velocity $v$ is the modulus of the velocity $\left(u_{x}, u_{y}\right)$.

In agreement with the predictions of the Hele Shaw theory (equations 11 and 12), the datapoints are fitted with a logarithmic law for the density and an $r^{-1}$ law for the velocity. The value of $Q$ used to fit the data is the one that is measured in the simulation: our injection boundary condition at a given pressure (density), fills the mould with a constant flow $Q$.

The above results show that Hele-Shaw model is correctly obeyed by the LB simulation.

\section{Simulation of a realistic moulding problem}

\subsection{Overview}

As a second sample application, we consider the moulding of a charge card with integrated chip. In this case, the space taken by the chip is excluded from the moulding domain.

The aim of this application is to show that even somewhat complex geometries can be addressed with the simulations. In general, the implementation of appropriate boundary conditions is a very delicate topic. However, fairly appealing results are obtained already with the simple boundaries we have implemented.

\subsection{Results}

In the simulation, the surface of the charge card is initially filled with air (at a density $\rho_{0}=1.0$ ). The polymer is injected on the side opposite to the chip, at a level corresponding to the middle of the chip location. 


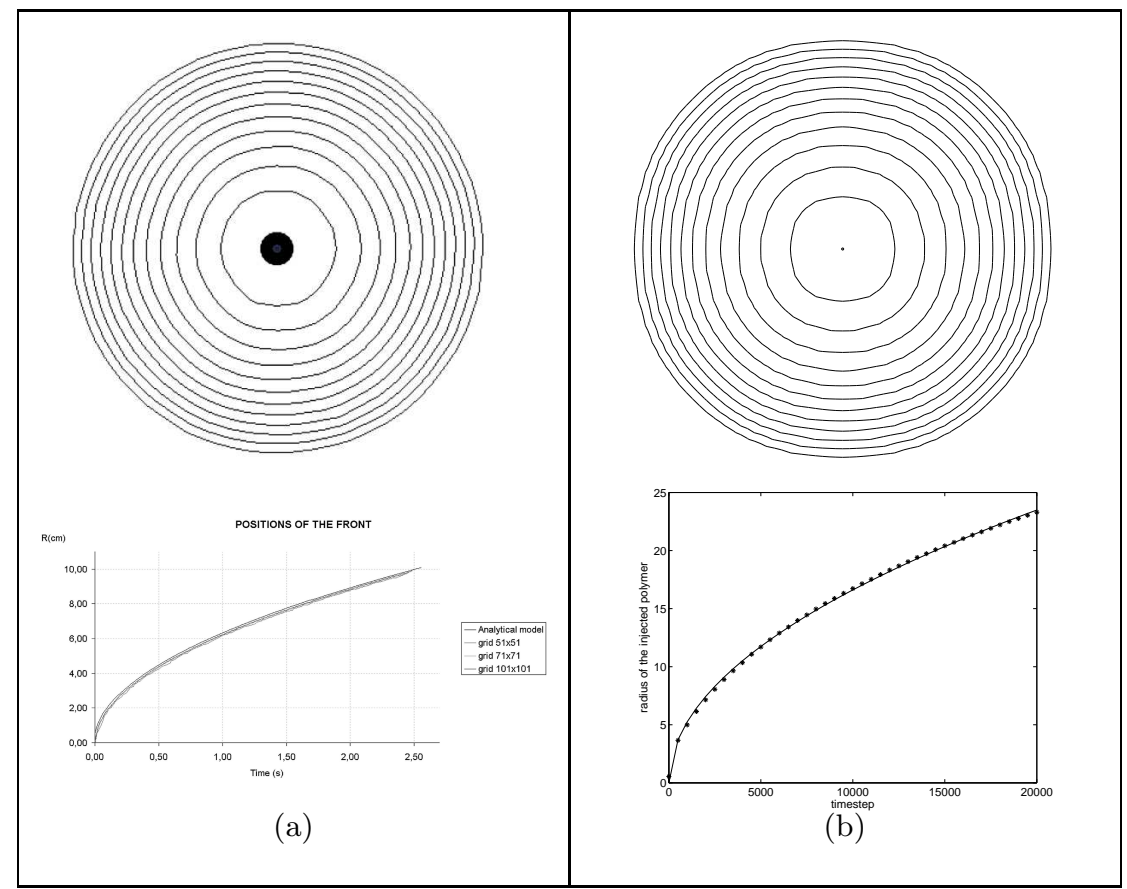

Fig. 3. Evolution of the disk radius during an injection in free space. On the left hand side (a), the results from the program MoldFlow are presented, on the right hand side (b) the results from a lattice Boltzmann simulation. On the upper part of the pictures, the surface of the injected polymer is plotted at successive timesteps. On the lower part, the curves for the time evolution of the radius are fitted with the predictions of the Hele-Shaw theory.

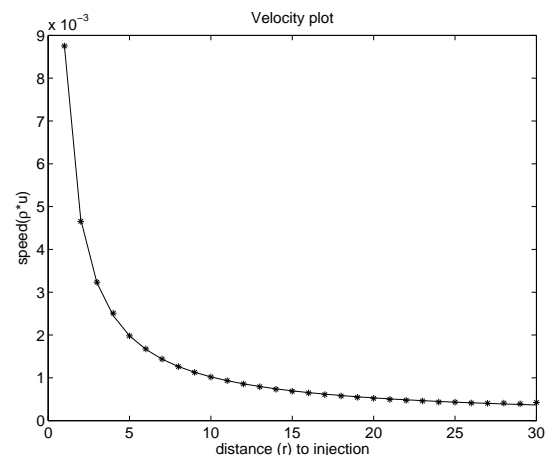

(a)

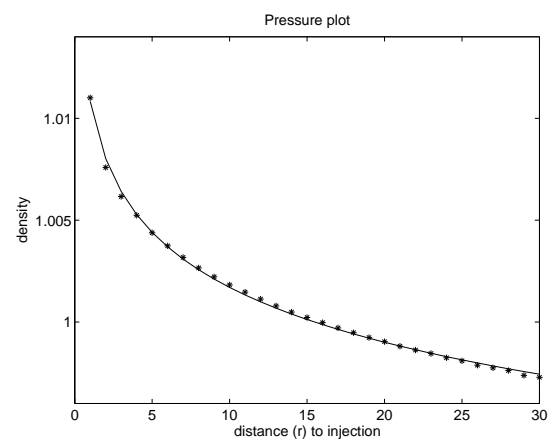

(b)

Fig. 4. Velocity profile (a) and pressure profile (b) at $t=140000$ obtained from the lattice Boltzmann simulation. The solid line shows a fit with the predictions of the Hele Shaw theory. 
The injection is made at a density $\rho_{i n}=1.01$. The air is evacuated on the right boundary of the card at a constant density $\rho_{\text {out }}=1.0$. All other boundaries implement a bounce-back condition with $g^{(0)}=g^{(1)}=0$, which produces a contact angle of $\pi / 2$. The surface tension is chosen at a value $W=-0.3$.

Figure 5 plots the area occupied by the polymer at successive timesteps, first calculated by the commercial program MoldFlow and, secondly, calculated by the LB method. The difference betweend (b) and (c) consists in the way the boundary conditions are defined on the chip perimeter (see caption). The results of both simulations are very similar in the beginning. In the second part of the simulation, the LB method turns out to be more sensitive to the geometry of the domain than the MoldFlow simulation.

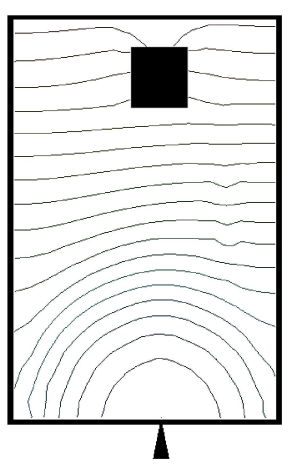

(a)

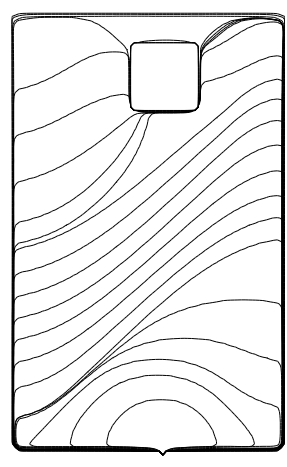

(b)

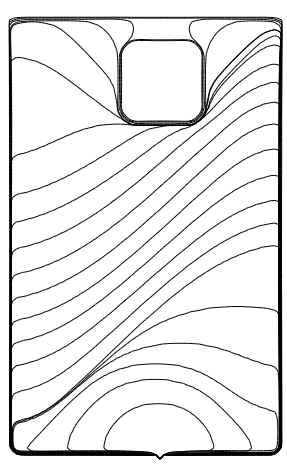

(c)

Fig. 5. Mould of a charge card. The curves show the propagation front of the polymer as computed from the program MoldFlow (a) and the lattice Boltzmann method (b,c). In (b), a bounce-back condition is applied on the surface of the chip, whereas in (c), the air is evacuated at constant density on the chip perimeter.

The sensitivity of the flow dynamics to the geometry of the domain that we observe in the LB simulation is due to the pressure of the air on the polymer. Indeed, one of the main advantages of the LB method, the ability to model the influence of the air on the dynamics, also introduces some complications. If, as in our simulations, the air is not evacuated fast enough, this can have a serious impact of the flow dynamics; the resulting flow geometry may disagree with real moulding applications, where the air is much lighter and less visous than the polymer. In this context, some work still needs to be done either on the flow properties of the air or on the boundary conditions. 


\section{Conclusion}

The LB methods brings several interesting elements in the process of injection modeling. The possibility to consider the full system of two fluids (air plus polymers) and to include air traps makes it possible to model how air escapes from the mould as the polymer expands. This feature is not offered by current softwares. In this respect, our preliminary results are relevant. They are especially encouraging in the case of the charge card with a complex geometrical shape.

One assumption of our preliminary study was that both fluids have the same viscosity and density. The simulation shows that although this assumption is sufficient for explaining some geometric properties of the moulded polymer, it is too strong to be able to represent dynamic features such as the detailed polymer front propagation profile. Therefore, future work might include a more realistic model for the different physical properties of the polymer and the air. Our study must be continued in order to give a reliable solution for polymer injection moulding and to include optimization processes where the best injection points and air-holes are determined to minimize weld lines formation.

In conclusion, we think that Lattice Boltzmann methods are promising candidates for the simulation of polymer injection moulding and for the solution of current problems such as melt polymer/mould wall interaction.

\section{References}

1. J. Serra. Image Analysis and mathematical Morphology, Vol. 1. Ac. Press, 1988.

2. M. Jourlin, G. Courbebaisse, and D. Garcia. Polymer molding simulation : A mathematical imaging approach based on propagation of discrete distances. Elsevier Computational Materials Sciences, 18(1):19-23, 1999.

3. G. Courbebaisse, D. Garcia, and P.Bourgin. A way towards optimization of injection molding. ASME Fluids Engineering Division Summer Meeting, 2003.

4. G.Aronsson. On p-harmonic functions, convex duality and an asymptotic formula for injection mold filling. European J. Applied Mathematics, 7:417-437, 1996.

5. Sauro Succi. The Lattice Boltzmann Equation, For Fluid Dynamics and Beyond. Oxford University Press, 2001.

6. N.S. Martis and H. Chen. Simulation of multicomponent fluids in complex 3d, geometries by the lattice Boltzmann method. Phys. Rev. E, 53:743-749, 1996.

7. JPH. Sergent J.F. Agassant, P. Avenas. La mise en forme des matières plastiques. Lavoisier, 1989.

8. Irina Ginzburg and Konrad Steiner. Lattice boltzmann model for free-surface flow and its application to filling process in casting. Journal of Comp. Physics, 185:6199, 2003.

9. B. Chopard and M. Droz. Cellular Automata Modeling of Physical Systems. Cambridge University Press, 1998.

10. Haiping Fang Lewen Fan and Zhifang Lin. Simulation of contact line dynamics in a two-dimensional capillary by the lattice boltzmann model. Physical Review E, 63(5):051603, april 2001.

11. Qisu Zou and Xiaoyi He. On pressure and velocity boundary conditions for the lattice botzmann bgk model. Phys. Fluids, 9(6):1591-1598, february 1997.

12. P. Kennedy. Flow analysis of injection molds. Hanser, 1995. 